\title{
On the Future of Biology
}

\author{
E.O. WILSON \\ Museum of Comparative Zoology, Harvard University, Cambridge, Massachusetts 02138 \\ Correspondence: ewilson@oeb.harvard.edu
}

\begin{abstract}
Biology is driven by two strategies of research. The first, using functional studies at the molecule-to-organism transitions, utilizes the principle that for every problem in biology, there is an organism ideal for its solution. The second, comparative and evolutionary in orientation, consists of thorough studies of every biological aspect of a given taxonomic group of species and follows the principle inverse to the first that for every organism, there is a problem to the solution for which it is ideally suited. The two approaches are complementary and of increased connectivity to each other. In passing from the traditional, intensely focused reductionist approach of 20th century biology to a more synthetic and authentically holistic phase, new emphasis will be placed on the major transitions between levels of biological organizations and onto processes of multilevel evolution by which they have been achieved.
\end{abstract}

The science of biology is coming together. Enough of its disciplines have been linked by cause-and-effect explanation to suggest how some of the framework of a unified biology can be visualized. At its foundation, biological knowledge conforms to two principles arguably firm enough to be called scientific laws, i.e., they are all-inclusive and with no proven exceptions, and their consequences can be followed wide and deep. The first law is that all of the entities and processes defining life are ultimately obedient to the laws of physics and chemistry. The second law, still in contention due to claims for counterexamples, is that all of the diagnostic entities and processes of life, and all of its diversity, have evolved by natural selection.

The conformity of life's processes to the physical sciences undergirds molecular and cellular biology. Its universal application has been challenged by the arguments for a supernatural intelligent design. Its champions may believe that scientists are in a conspiracy against intelligent design, but the opposite is true. Every researcher would like to discover an extrasomatic force: This would be one of the greatest achievements in the history of science. The problem is that not a trace of evidence has ever been found, nor has the need for such a theoretical place marker arisen from any database. The confidence of biological researchers in the complete physicality of life remains adamantine.

The same can be said for the exclusive role of natural selection in genetic evolution. The ruins of alternative theories, from neo-Lamarkian to punctuated equilibrium, litter the archives of past decades. The mysterious forces that they imply, undenoted and extraneous to the empirical evidence of genetics, do not aid evolutionary theory. Modern evolutionary theory, rooted in molecular genetics, begins with the distinction between the units of heredity and the targets of natural selection. The units are the genes, respectively, the base-pair sequences of their codes, their degree of duplication in the genome by tandem repeat sequencing, their location in the genome, and the interaction of their products during epigenesis. The targets of natural selection are the heritable phenotypes prescribed by the genes, including the plasticity of response in the final products composing the phenotypes.

Natural selection is multilevel: A phenotypic target can exist at any level of biological organization, from macromolecules to chromosomes to eukaryotic cells to multicellular organisms, and onto organized social groups, populations of organisms and groups, and finally, arguably, entire ecosystems.

Other agents have been found that can drive evolution for very short distances, but they are neither creative in shaping adaptation to the environment nor significant in magnitude to compare with natural selection. Different rates of mutation between opposing alleles can shift allelic frequencies, but the effect is easily overwhelmed by even barely detectable degrees of selection. Similarly, genetic drift, when permitted by low selection pressures and small population size, can shift frequencies, but it tends to lose alleles, narrowing the potential for future evolution.

Multiple selection is subject to reverberation in its impact. When selection favors one form of heritable trait at any given level of biological organization, it favors or disfavors traits prescribed by the same allele (or ensemble of alleles) at other levels. A familiar example is the inherited predisposition to certain kinds of cancer: An allele predisposing the unregulated growth of cells is favored at the level of the cell carrying it but disfavored by the cancer caused at the level of the organism. Organisms with cancer are hosts to multitudinous healthy offspring of the original mutant cell, yet they lose in competition with healthy organisms lacking the mutant allele.

Multilevel selection typically places selection at adjacent levels in opposition. To achieve a new level, for example, a multicellular organism or a complex society, requires some degree of altruism on the part of individuals at the lower level. It is promoted by group selection and opposed by individual-level selection. Such major transitions across levels of organization have very rarely 
occurred in evolution and taken long periods of geological time to be achieved even once. The origin of the eukaryotic organisms took more than half the known history of life to be attained, from the beginning more than 3.5 billion to 1.5 billion years ago. Multicellular animals were not added for more than another half-billion years. The origin of insect superorganisms, in particular the tightly knit colonies of ants and termites or their equivalent, was not attained during the first great insect adaptive radiation in the late Paleozoic Era, 350-250 years before the present, and occurred only well into the late Jurassic to very early Cretaceous periods 200-140 million years ago.

It is in the major transitions of evolution that biology will more predominantly dwell. The Age of Reduction in biology has largely passed. Although enormous amounts of new information will continue to be yielded by the cleavage and analysis of systems, the big problems in each discipline are those that require an exactitude of synthesis or, more accurately, resynthesis of reduced systems. As more biological systems are understood, and with them the major transitions, common principles of emergent evolution should become apparent. There appears to be no a priori way to create such a unified theory of biology at the present time. It awaits far more empirical information than we now possess, requiring immense amounts of hard work with real organisms.

Modern biology has long been addressed by two very different strategies of research. The first strategy follows the dictum that for every problem in biology, there exists an organism ideally suited to solve it. The bacterium Escherichia coli has thus triumphed for molecular genetics, the nematode Caenorhabditis elegans for the neuronal basis of behavior, the honeybee for the instinct and self-organization of animal societies, and human beings for the conscious mind.

If those faithful to this dictum are called the problem solvers, the second tribe may be called the naturalists. The research strategy of the naturalists is the inverse of the strategy of the problem solvers. It is that for every organism, there exists a problem for which the organism is ideally suited. The procedure of the naturalist is to adopt a group of species, such as conifers, diatoms, and orbweaving spiders, and learn as much as possible about the group across all levels of biological organization.

The overarching goal of the scientific naturalist is the complete mapping of biodiversity while acquiring knowledge of it at each level of biological organization. The daunting nature of this task is made clear when an effort is made to estimate even roughly the total number of species on Earth. When the hyperdiverse microorganisms, fungi, and small invertebrates are put in the roster, it turns out that we have discovered and named probably $10 \%$ or fewer of the species living on Earth. The variety of genes prescribing all of them is astronomical and, of course, even less known.

The third great goal, yet even more daunting, is the history of all life. Because of the paucity of information on the living species and their genomes, the phylogenies of only a few of the best-known groups, such as birds and flowering plants, can be drawn with any confidence. And to a record of all that has happened in past ages, the infant discipline of paleobiology faces a journey likely to take centuries, if not millennia, and is likely never to end.

Problem solvers, at work mostly in the laboratory, explain the proximate causes of biological phenomena, usually those visible only at a microscopic level - in other words, how the system works. Naturalists, working typically in the field but also experimentally in the laboratory, stress the adaptation of biological phenomena to the environment, the why in its attempted full explanation.

Although the two approaches have seemed at times to represent independent cultures, they are in fact complementary and can with more information be fitted nicely together. The boundaries are in fact being erased. Scientific naturalists, as organismic and evolutionary biologists, use the methods of molecular and cellular biology, whereas molecular and cellular biologists grow more prone to address patterns of diversity and evolution. With increasing frequency, the two tribes collaborate in research projects. At the risk of oversimplification, it can be said that the naturalists discover the problems in nature that the problem solvers solve.

The trajectory of a unified biology can be visualized to be $\mathrm{T}$ shaped. The horizontal arm is biodiversity at the level of species and genetic strains within each species. One vertical arm is the model species of the problem solver, reaching from the populations that constitute the species or strains down through the levels of biological organization, from the ecosystems of which it is a part to the genes that prescribe its diagnostic traits. At present, although fewer than 100 such model species are under such scrutiny, in a decade or two, there will be thousands and then tens of thousands. The process will accelerate as DNA sequencing grows ever faster and cheaper. Existing knowledge will be immediately available and its growth followed in real time, as the Encyclopedia of Life gears up to provide complete information through a single portal for all species known.

The more the two-dimensional array of knowledge is filled in, the more the gaps in knowledge and the most promising new directions of research will become apparent. At the same time, paleobiology and phylogenetic analyses will add the dimension of time. A unified biology is a goal worth thinking about, even if still far from within our grasp. Biologists are stretching causal explanations across wider segments of the levels of biological organization and in broader arrays of species. In so doing, they are melding explanations of function and adaptation, as well as molecules and cells with organisms and species. As a result, the emphasis in research is shifting from one or two levels of biological organization to all of the transitions between levels.

The major questions of biological theory are those of systems biology. They take the following form: How do the elements and processes that define one level emerge from the level below it? And why, and under what circumstance? Then, were the evolutionary routes the only ones, and if not, were they at least the optimal ones?

A unified biology will be the one that maps the pathways from molecules to ecosystems in unbroken transits of causal explanation. It will disclose the still unimagined commonalities, if such exist, among the evolutionary transitions. It will also provide insight into whatever different genetic codes and rules of transitions are possible, and even which of these might exist in other worlds. 


\section{$\$_{\mathrm{CSH}}^{\infty} \&$ Cold Spring Harbor Symposia SYMPOSIA on Quantitative Biology}

\section{On the Future of Biology}

E.O. Wilson

Cold Spring Harb Symp Quant Biol 2009 74: 9-10 originally published online October 20, 2009 Access the most recent version at doi:10.1101/sqb.2009.74.020

\section{License}

Email Alerting Receive free email alerts when new articles cite this article - sign up in the box at the Service top right corner of the article or click here. 